\title{
Transport of organic solutes in ion-exchange membranes: Mechanisms and influence of solvent ionic composition
}

\author{
Lingshan Ma a Leonardo Gutierrez ${ }^{\mathrm{a}, \mathrm{b}}$, Rhea Verbekec ${ }^{\mathrm{c}}$, Arnout D'Haese , \\ Muhammad Waqas a, Marcel Dickmann ${ }^{\text {, }}$, Ricardo Helm d, Ivo Vankelecom ${ }^{c}$, \\ Arne Verliefde ${ }^{a}$, Emile Cornelissen ${ }^{\mathrm{a}, \mathrm{e}, *}$ \\ a Particle and Interfacial Technology Group, Ghent University, Belgium \\ b Facultad del Mar y Medio Ambiente, Universidad del Pacifico, Ecuador \\ ${ }^{\mathrm{C}}$ Membrane Technology Group, Centre for Membrane Separations, Adsorption, Catalysis and Spectroscopy for Sustainable Solutions, KU Leuven, Belgium \\ ${ }^{d}$ Institut für Angewandte Physik und Messtechnik, Universität der Bundeswehr München, Germany \\ e KWR Water Research Institute. Netherlands
}

\section{A R T I C L E I N F O}

\section{Keywords:}

Ion-exchange membranes

Organic solutes

Transport mechanism

Selective separation

Influence of salts

\begin{abstract}
A B S T R A C T
Ion-exchange membrane (IEM)-based processes are used in the industry or in the drinking water production to achieve selective separation. The transport mechanisms of organic solutes/micropollutants (i.e., paracetamol, clofibric acid, and atenolol) at a single-membrane level in diffusion cells were similar to that of salts (i.e., diffusion, convection, and electromigration). The presence of an equal concentration of salts at both sides of the membrane slightly decreased the transport of organics due to lower diffusion coefficients of organics in salts and the increase of hindrance and/or decrease of partitioning in the membrane phase. In the presence of a salt gradient, diffusion was the main transport mechanism for non-charged organics, while the counter-transport of salts promoted the transport of charged organics through electromigration (electroneutrality). Conversely, the co-transport of salts hindered the transport of charged organics, where diffusion was the main transport mechanism of the latter. Although convection played a role in the transport of non-charged organics, its influence on the charged solutes was minimal due to the dominant electromigration. Positron annihilation lifetime spectroscopy showed a bimodal size distribution of free-volume elements of IEMs, with both classes of free-volume elements contributing to salt transport, while larger organics can only transport through the larger class.
\end{abstract}

\section{Introduction}

Industrial wastewaters (e.g., food processing, beverage, textile, petroleum, and pharmaceutical) can be streams of complex physicochemical characteristics, often containing a wide range of complex constituents (e.g., aromatic hydrocarbons, dyes, oil, heavy metals, pharmaceuticals, pesticides); thus, posing an environmental threat to natural waters (Al-Amshawee et al., 2020; Lefebvre and Moletta, 2006; Zhao et al., 2019). The co-existence of high salinity and high concentrations of organic solutes in some of these wastewaters considerably limits the performance of physicalchemical and biological treatments. For example, conventional activated sludge systems are negatively influenced by high salinity (Chen et al., 2018); while other treatments (e.g., coagulation-

\footnotetext{
* Corresponding author.

E-mail addresses: lingshan.ma@ugent.be (L. Ma), Emile.Cornelissen@UGent.be (E. Cornelissen).
}

flocculation) have been reported as inefficient for removing emerging contaminants in wastewater, leading to the release of organic micropollutants (OMPs, ng $\mathrm{L}^{-1} \sim \mu \mathrm{L} \mathrm{L}^{-1}$ range) into aquatic environments (Grandclement et al., 2017). Therefore, selective separation of salts and organic solutes is often needed in these wastewaters to improve their treatability, as well as increase potential resource recovery. Ion-exchange membrane (IEM)-based processes could theoretically have the potential to selectively separate organic and inorganic solutes from industrial wastewaters, given the different physicochemical characteristics of these solutes - and their different mobility in IEM. However, more insight into the behavior of organic solutes in IEM processes is still needed, especially under the influence of solvent ionic composition.

IEMs have been successfully used in several electrodialysis (ED)-based processes dealing with complex streams in the field of resource recovery, such as the recovery of lithium from brines, salts from urine, and nutrients from waste streams (Pramanik et al., 2020; Pronk et al., 2006; Ward et al., 2018). 
ED-based processes have also been used in the production, purification, and softening of drinking water (Campione et al., 2018; Gonzalez et al., 2017; Strathmann, 2010). Besides ED, other emerging IEM-based technologies are being studied. Reverseelectrodialysis (RED), for example, has been tested as a predesalination step for seawater reverse-osmosis and for generating energy based on the salinity gradient between seawater/brines and impaired wastewater (Li et al., 2013; Nam et al., 2019; Vanoppen et al., 2019). However, there is still a lack of fundamental knowledge on the transport of organic solutes/OMPs in IEMs in complex streams (i.e., salt-containing solutions); thus, raising concern on the contamination of the recovered products and drinking water source.

Despite recent research efforts, there is yet no consensus on the transport mechanisms of organic solutes/OMPs in IEMs in saltcontaining solutions. Briefly, Arola et al. (2019) suggested that the transport of pharmaceuticals in IEMs during nutrient concentration in ED was mainly influenced by the hydrophobicity/hydrophilicity and electrostatic interactions of the solutes and membranes. Roman et al. (2019) observed that the transport of negativelycharged and non-charged OMPs $\left(100 \mu \mathrm{g} \mathrm{L}^{-1}\right)$ in IEMs under RED conditions was driven by Donnan dialysis and diffusion, respectively, while positively-charged OMPs were barely transported. Additionally, a previous investigation by Vanoppen et al. (2014) on the transport of 16 OMPs ( $\left.100 \mu \mathrm{L} \mathrm{L}^{-1}\right)$ in IEMs during ED suggested electrostatic interactions dominated the transport of charged solutes, while the external current exerted a minimal effect. Moreover, salts hindered the transport of charged OMPs regardless of the concentration of salts in the concentrate and diluate, while water and salt transport showed an insignificant impact on the transport of non-charged OMPs. In contrast, Han et al. (2016) suggested convection as a key transport mechanism for non-charged organic solutes (undissociated acetic acid, phenol, glucose) in saltcontaining solutions $\left(\mathrm{NaCl}, \mathrm{MgCl}_{2}, \mathrm{Na}_{2} \mathrm{SO}_{4}\right)$ under ED. In singlemembrane level experiments, Ma et al. (2018) showed that diffusion was the main transport mechanism of non-charged organic solutes (i.e., paracetamol, theophylline, and phenazone) in anionand cation-exchange membranes (AEMs and CEMs) in the absence of salts, and physically described the transport by the solutiondiffusion model. Noteworthy, the majority of the studies in the literature have been designed around complex stack configurations containing multiple pairs of AEMs and CEMs, different types of salts, flowrates, and current densities, while the investigations at the single-membrane level are limited. Moreover, these studies mainly focused on the interfacial interactions occurring between solute and membrane, while the dominant transport mechanism of the organic solutes/OMPs under different conditions (e.g., absence/presence of salts) is still under debate. Therefore, a single membrane configuration could highly assist in ultimately understanding the transport mechanisms of organic solutes in IEMs and the influence of salts on the transport.

In addition, the transport mechanisms of solutes in IEMs have been quantitatively considered in current models, where inorganic salts were used for the model development and validation (Fan and Yip, 2019; Kingsbury and Coronell, 2020; Tedesco et al., 2016, 2017). Although the transport mechanisms of organic solutes would be similar to those of salts (i.e., diffusion, convection, and electromigration), only a few studies have tackled this gap in knowledge; thus, this topic remains under debate. Also, given the industrial need for selective separation of organic and inorganic solutes in IEM-based technologies, organic and inorganic solutes are regarded as two types of species. The influence of solvent ionic composition on the transport mechanism of the organic solutes, thus, is of high value and lacks research.

In this study, the transport of organic solutes in commercial AEMs and CEMs in salt-containing solutions was investigated at a single-membrane level. Three pharmaceuticals (i.e., paracetamol, clofibric acid, and atenolol) were selected based on their worldwide use, high environmental relevance, and their different physicochemical properties (e.g., size, hydrophobicity, charge). The influence of salts in solution on a) the physicochemical properties of the organic solutes, b) the transport of organic solutes in IEMs, and c) water transport through the IEMs were comprehensively studied. The experimental conditions were carefully designed to mimic ED-based processes (e.g., emerging RED technology). Thus, three transport mechanisms of organic solutes in IEMs were distinguished (i.e., diffusion, convection, and electromigration). For the first time, positron annihilation lifetime spectroscopy (PALS) was used to investigate the size and abundance of free-volume elements of AEMs and CEMs, which highly assisted in understanding the role of membrane parameters in the transport of organic solutes and salts. This study provides essential knowledge on the transport of organic solutes in IEM-based processes in saline environments, ultimately leading to a more efficient selective separation of organic/inorganic solutes in ED-based systems, and to the future design of highly selective next-generation IEMs.

\section{Materials and methods}

\subsection{Selection and characterization of organic solutes}

Paracetamol, clofibric acid, and atenolol were selected as model non-charge, negatively-charged, and positively-charged organic solutes, respectively. The diffusion coefficient of the organic solutes in Milli-Q water, $0.1 \mathrm{~g} \mathrm{~L}^{-1}$, and $29.2 \mathrm{~g} \mathrm{~L}^{-1} \mathrm{NaCl}$ solutions was measured by ${ }^{1} \mathrm{H}$ NMR (Proton Nuclear Magnetic Resonance) as previously described (Ma et al., 2018). The radii $\left(r_{s}\right)$ of the organic solutes were calculated by the Stokes-Einstein equation. The characteristics of the organic solutes are listed in the supplementary information (SI) section (Table S1).

\subsection{Characterization of ion-exchange membranes}

The experiments were conducted using commercial homogeneous AEM and CEM. The thickness and contact angle of the membranes was measured by a screw micrometer and a KRÜSS Drop Shape Analyzer, respectively, with a minimum of 10 measurements per coupon. The protocols for determining the swelling degree and water permeability are described in sections S2-3.

Positron annihilation lifetime spectroscopy (PALS) is a nondestructive technique to probe free-volume elements in porous materials, such as polymeric membranes. Its principles are described in section S4. PALS measurements under vacuum conditions were performed on the AEM and CEM with the pulsed low energy positron beam system (PLEPS) at the neutron-induced positron source Munich (NEPOMUC, FRM-II reactor in Garching, Germany) (Egger et al., 2007; Hugenschmidt et al., 2008; Hugenschmidt et al., 2012; Sperr et al., 2008). Positron implantation energies between 1 and $18 \mathrm{keV}$ were used under ultra-high vacuum conditions to investigate the free-volume element depthprofiles of the IEMs. The implantation depths at each energy were calculated based on a well-established procedure (Algers et al., 2003). Due to limitations in the maximum achievable implantation energy of the used PALS setup ( $18 \mathrm{keV}$ ), information on the size and intensity of free-volume elements originating solely from the upper $\sim 3 \mu \mathrm{m}$ of the membrane (i.e., from the surface up to $3 \mu \mathrm{m}$ inside the membrane) was obtained. However, IEMs are known to be dense and homogeneous membranes, where heterogeneities that can result from the synthesis procedure would most probably arise at the surface. Deeper inside the membrane and especially down to $3 \mu \mathrm{m}$, these surface effects are absent, and the information obtained originates from the bulk of the membrane. 


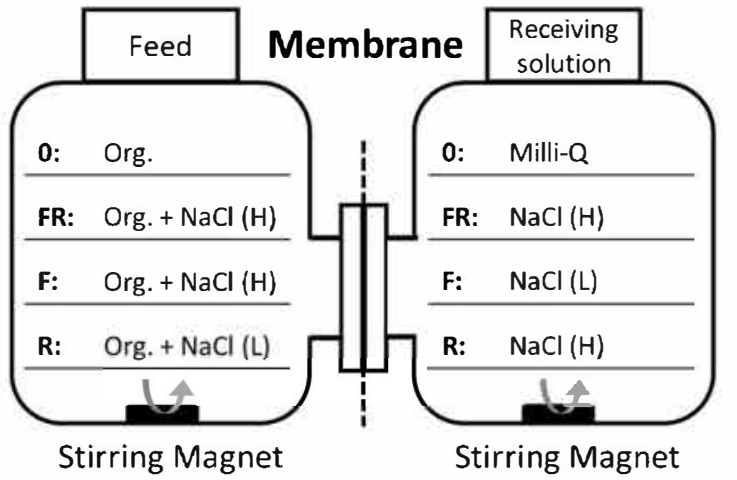

- Org. (Organic solutes) - $\mathrm{NaCl}(\mathrm{H}): 29.2 \mathrm{~g} \cdot \mathrm{L}^{-1}$ - $\mathrm{NaCl}(\mathrm{L}): 0.1 \mathrm{~g} \cdot \mathrm{L}^{-1}$

Fig. 1. Composition of the solutions in the feed and receiving solution compartments of the diffusion cell setup under conditions $0, F R, F$, and $R$.

Thus, it can be substantiated that the material properties remain constant in increased depths and that the distribution and size of free-volume elements obtained in the upper $3 \mu \mathrm{m}$ are representative throughout the complete thickness of the membrane. For each spectrum, 4 million counts were collected at a rate of $\sim 10,000$ cts $\mathrm{s}^{-1}$ and a time resolution of $\sim 250 \mathrm{ps}$. The measured spectra were deconvoluted from the background signal and the instrument resolution function, the latter being determined by measuring p-doped $\mathrm{SiC}$, a standard sample with well-known lifetimes. All spectra were analyzed with PALSfit3 software (Kirkegaard et al., 2017). The bestfitting results with the simplest multi-exponential model required four lifetime components ( $p$-Ps, free $e^{+}, 0-\mathrm{Ps}_{\text {short }}\left(\tau_{3}\right)$, and $0-\mathrm{Ps}_{\text {long }}$ $\left.\left(\tau_{4}\right)\right)$. The overall fit variances were below 1.2 for all samples.

\subsection{Experimental setup and protocol}

Experiments with paired diffusion cells at a single-membrane level were conducted to study the relative impact of diffusion, convection, and electromigration (i.e., being induced by a concentration gradient, bulk transport of water molecules due to osmotic pressure, and electrostatic potential gradient, respectively) on the overall transport of organic solutes in AEMs and CEMs. Briefly, a flat AEM or CEM (active area: $5.94 \mathrm{~cm}^{2}$ ) was placed between the two parts of a diffusion cell (capacity $150 \mathrm{~mL}$ each) and connected by a clamp. The resulting two compartments were termed as feed (containing organic solutes, denoted as Org.) and receiving solution (Fig. 1). A high concentration (i.e., $29.2 \mathrm{~g} \mathrm{~L}^{-1}$, mimicking industrial effluent or seawater) and a low concentration (i.e., $0.1 \mathrm{~g}$ $\mathrm{L}^{-1}$, mimicking impaired water or freshwater) of $\mathrm{NaCl}$ were used and termed as $\mathrm{NaCl}(\mathrm{H})$ and $\mathrm{NaCl}(\mathrm{L})$, respectively. No external current was applied in this experimental setup. Therefore, the driving force of electromigration (i.e., electrostatic potential gradient) in this study was only triggered spontaneously by the transport of counter-ions while co-ions are rejected. The experiments were conducted for $24 \mathrm{~h}$, where at least six samples were collected to study the trend in the transport of organic solutes from the feed to the receiving solution. The concentration of organic solutes was measured by UV-spectrophotometry (VWR UV-1600 PC) at specific wavelengths (Table S1). The fluxes ( $\left.\mathrm{J}, \mathrm{mol} \mathrm{m}^{-2} \mathrm{~h}^{-1}\right)$, permeability constants $\left(B, \mathrm{~m} \mathrm{~s}^{-1}\right)$, and the percentage of the total transported mass (\%) of organic solutes were calculated after $24 \mathrm{~h}$. The water transport, temperature, and $\mathrm{pH}$ were continuously monitored throughout all the experiments.

Four experimental conditions were tested, in which the composition of the feed and receiving solutions were varied (Fig. 1) as follows: 1) condition 0: organic solutes in the feed, and Milli-Q water in both feed and receiving solution, 2) condition FR: organic solutes in the feed, and $\mathrm{NaCl}(\mathrm{H})$ in both feed and receiving solution, 3) condition $\mathbf{F}$ : organic solutes $+\mathrm{NaCl}(\mathrm{H})$ in the feed, and $\mathrm{NaCl}(\mathrm{L})$ in the receiving solution, and 4) condition R: organic solutes $+\mathrm{NaCl}(\mathrm{L})$ in the feed, and $\mathrm{NaCl}(\mathrm{H})$ in the receiving solution. Due to their low solubility in water, the initial concentration of clofibric acid and atenolol in the feed $\left(\Delta C_{0}\right)$ was $0.1 \mathrm{~g} \mathrm{~L}^{-1}$. In contrast, the initial concentrations of paracetamol in the feed were $0.1,1$, and $2 \mathrm{~g} \mathrm{~L}^{-1}$ for each condition. Conditions 0 and FR would provide information on the diffusive transport and electromigration of non-charged and charged organic solutes, respectively, in the absence and presence of salts in both the feed and receiving solution. Conditions $\mathrm{F}$ and $\mathrm{R}$ were conducted to elucidate the influence of the a) salt concentration gradient and b) transport direction of salts (i.e., from feed to receiving solution or vice-versa) on the transport mechanisms of organic solutes (i.e., diffusion, convection, and electromigration).

To achieve a negative charge for clofibric acid $(\mathrm{pH}>6)$ and a positive charge for atenolol $(\mathrm{pH}<6)$, the $\mathrm{pH}$ of the clofibric acid- and atenolol-containing solutions was adjusted to approximately 6.2 and 5.1 using $\mathrm{NaHCO}_{3}$ and $\mathrm{HCl}$, respectively. The $\mathrm{pH}$ of the solutions was continuously monitored during the experiments, where no further $\mathrm{pH}$ adjustment was necessary. All membranes were saturated with organic solutes before experiments, as previously described (Ma et al., 2018). Briefly, all the membranes were soaked into solutions containing the organic solutes of interest (at the concentrations of interest) for $24 \mathrm{~h}$, followed by Milli-Q water rinsing, and finally soaked into Milli-Q water for $10 \mathrm{~h}$. Additional control experiments were conducted to study a) adsorption and desorption of positively-charged atenolol in CEM, and b) the impact of salts on the desorption of organic solutes from the saturated membranes (protocols described in sections S5-6).

\section{Results and discussion}

\subsection{Characterization of organic solutes and ion-exchange membranes}

The diffusion coefficient of the selected organic solutes in water showed an inverse correlation with their molecular weight, and followed the following order: paracetamol $\left(7.5 \times 10^{-10} \mathrm{~m}^{2} \mathrm{~s}^{-1}\right)>$ clofibric acid $\left(6.70 \times 10^{-10} \mathrm{~m}^{2} \mathrm{~s}^{-1}\right)>$ atenolol $\left(5.38 \times 10^{-10} \mathrm{~m}^{2} \mathrm{~s}^{-1}\right)$. When salts were present, the diffusion coefficient of the pharmaceuticals decreased due to an increase in viscosity of the solvent (Table S1) (Hayduk and Laudie, 1974).

The characteristics of AEM and CEM are shown in Table S2. The thickness of AEM was lower than CEM $(114 \mu \mathrm{m}$ and $127 \mu \mathrm{m}$, respectively), while the contact angle was higher for the AEM (57 ${ }^{\circ}$ and $18^{\circ}$, respectively). The permselectivity of AEM and CEM was $91 \%$ and $97 \%$, and the average swelling degree soaked in MilliQ water was $48 \%$ and $35 \%$, respectively. The swelling degree of membranes would depend on their cross-linking degree and crystallinity (Gugliuzza, 2015). The lower swelling degree of CEMs indicates a higher cross-linking degree, which may lead to a lower transport of organic solutes compared to AEMs (Długołęcki et al., 2008; Strathmann, 2004; Zhang et al., 2009; Ma et al., 2018). The water permeability of AEMs and CEMs showed statistically similar values (two-tailed t-test, $P>0.05$ ) of 10.6 and $11.0 \mathrm{~mL} \mathrm{bar}^{-1} \mathrm{~m}^{-2}$ $\mathrm{h}^{-1}$, respectively. Therefore, the transport of water in these IEMs would be expected to be similar under the same osmotic pressure. The results of the two control experiments (i.e., saturation and desorption of organic solutes) are discussed in sections S5 and S6, respectively. Briefly, the desorption of the organic solutes from the saturated membranes was negligible and did not impact their transport (Fig. S1-4).

The average size and abundance of the free-volume elements of AEM and CEM were determined using PALS under vacuum 

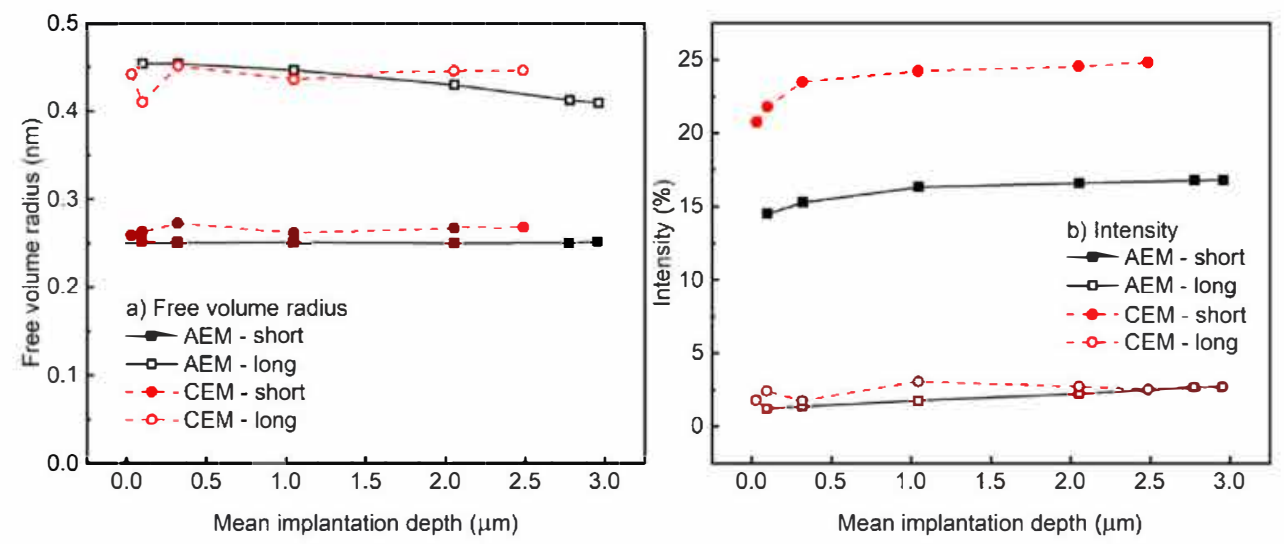

Fig. 2. (a) free-volume radius and (b) intensity as a function of mean implantation depth of AEM and CEM measured by PALS. Short and long correspond to o-PS ${ }_{\text {short }}$ and $o-P s_{\text {long, }}$ two classes of smaller and larger free-volume elements, respectively.

conditions (Fig. 2). Both AEM and CEM showed a bimodal pore size distribution, i.e., containing two classes of free-volume elements (Fig. 2a). 0 -Ps short corresponds to a class of smaller freevolume elements with an average radius of $0.25 \mathrm{~nm}$ and $0.27 \mathrm{~nm}$

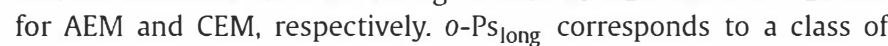
larger free-volume elements with an average radius of $0.43 \mathrm{~nm}$ and $0.44 \mathrm{~nm}$ for AEM and CEM, respectively. PALS results showed that the free-volume element radius of AEM and CEM under vacuum conditions do not significantly differ for both classes of pore sizes. However, since the free-volume elements were measured in a dry (non-swollen) state, the swelling degree of both membranes was considered by multiplying it with the free-volume element radius. The calculated free-volume element radius of AEM and CEM in solution would be estimated as $0.37 \mathrm{~nm}$ and $0.36 \mathrm{~nm}$ for 0 -Ps short (i.e., similar for both membranes), and $0.64 \mathrm{~nm}$ and $0.59 \mathrm{~nm}$ for

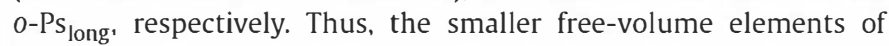
both IEMs are large enough to transport $\mathrm{Na}^{+}$and $\mathrm{Cl}^{-}$in both the crystal and (partially) hydrated state, given their hydrated radius of $0.18 \mathrm{~nm}$ and $0.20 \mathrm{~nm}$, respectively, reported by (Nghiem et al., 2006). The osmotic deswelling of both AEM and CEM in $29 \mathrm{~g} \mathrm{~L}^{-1}$ $\mathrm{NaCl}$ solutions was negligible for both types of the membranes, thus, exerting no influence on the free-volume elements. Additionally, the free-volume profiles of AEM and CEM as a function of membrane depth (i.e., starting at the surface and moving deeper inside the membrane) are almost constant (coefficient of variation $<5 \%$ ), indicating that both IEMs have free-volume elements of uniform size throughout the membrane. Likewise, the intensity of the 0 -Ps signal is relatively constant with increasing mean implantation depth for both AEM and CEM, again substantiating a considerably uniform morphology along the membrane thickness (Fig. 2b). The slight increase of the intensity at the membrane surface $(<0.5 \mu \mathrm{m})$ is due to the positron surface and interface effects (as explained in Section 2.2). The considerably higher intensities of the 0 -Ps short $_{\text {sh }}$ AEM $(\sim 16 \%)$ and CEM $(\sim 23 \%)$ compared to 0 -Ps ( $2 \%$ of both AEM and CEM) indicate that the small free-volume elements are more abundant than the larger ones in both membranes. When comparing AEM with CEM, the higher intensity of the $0-\mathrm{Ps}_{\text {short }}$ of CEM $(\sim 23 \%)$ would indicate a higher abundance of free-volume elements of this class compared to AEM $(\sim 16 \%)$. However, as AEM and CEM contain different functional groups which might differently affect the 0 -Ps formation probability, no conclusion can be drawn on the abundancy of free-volume elements for the different types of membranes based on the intensity results. Complementary information (e.g., membrane backbones and functional groups) is thus needed to elucidate whether CEM contains a higher fraction of free-volume elements compared to AEM.

\subsection{Transport of organic solutes in the absence of a salt gradient}

\subsubsection{Absence of salts in solution in both compartments of the diffusion cell}

The diffusive transport of organic solutes was studied under the absence of salts in both compartments of the diffusion cells (i.e., condition 0 ). The concentration gradient-based transport of noncharged organic solutes in IEMs in the absence of osmotic pressure and current has previously been shown as purely diffusiondriven (Ma et al., 2018). In this study, the diffusive transport of non-charged paracetamol in AEM and CEM was clearly evidenced by the direct proportionality of solute fluxes to the concentration difference between both solutions, for different initial concentration differences and over the course of the entire experiment (Fig. 3a and 3b). The total mass of the organic solutes in the feed and receiving solution did not significantly change during $24 \mathrm{~h}$ for both AEM and CEM (Fig. 3a), indicating no adsorption of paracetamol onto the membranes. The transport of paracetamol through the CEM was lower than for the AEM. This lower transport of organic solutes in CEM has been previously explained by its higher cross-linking degree, which was suggested to lead to lower porosity and average free-volume (i.e., both influencing the transport of organic solutes) (Ma et al., 2018; Vanoppen et al., 2014). Although paracetamol (radius of $0.32 \mathrm{~nm}$ ) would be potentially transported through both classes of free-volume elements in the two IEMs, the higher transport of paracetamol in AEM could be due to 1) the larger $0-\mathrm{Ps}_{\text {long }}$ free-volume element radius considering its swelling degree (i.e., lower size exclusion), 2) the lower cross-linking degree (could be indicated by higher swelling degree), and 3 ) the lower thickness of AEMs.

The transport profile of negatively-charged clofibric acid in AEMs at a $\Delta C_{0}=0.1 \mathrm{~g} \mathrm{~L}^{-1}$ was not linear, as the transport stopped after $18.5 \mathrm{~h}$ (Fig. 3c). The concentration of the receiving solution only reached $17.2 \mathrm{mg} \mathrm{L}^{-1}$ after $24 \mathrm{~h}$ and was far from equilibrium of the concentration difference across the membrane (i.e., $50 \mathrm{mg}$ $\mathrm{L}^{-1}$ at each compartment, assuming no adsorption phenomena). The mass balance showed negligible adsorption after $24 \mathrm{~h}$. Due to the similar radius of clofibric acid $(0.36 \mathrm{~nm})$ to the $0-\mathrm{Ps}_{\text {short }}$ freevolume elements $(0.37 \mathrm{~nm}$ considered swelling degree), its transport would mostly occur through 0 -Ps $s_{\text {Iong }}$ free-volume elements. This non-linear profile and concentration plateau far from equilibrium indicated that the transport of clofibric acid was not purely driven by concentration gradient; thus, the electrostatic potential gradient would have played a key role. Unlike non-charged organic solutes, charged organic solutes transport through IEMs as ions. The transport of negatively-charged organic solutes would generate a charge gradient across the membrane. To follow the 

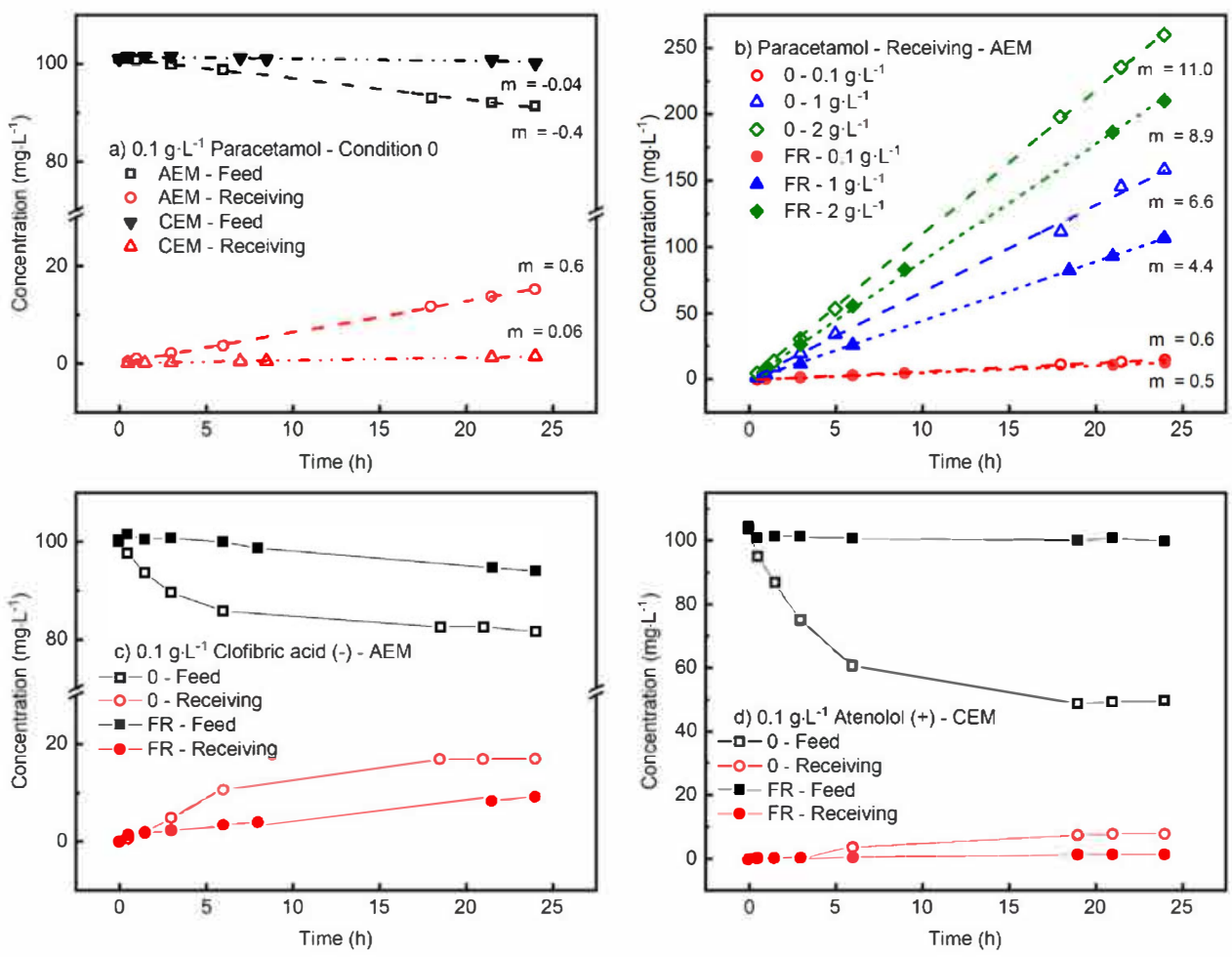

Fig. 3. Transport profile of (d) paracetamol in AEM and CEM $\left(\Delta \mathrm{C}_{0}=0.1 \mathrm{~g} \mathrm{~L}^{-1}\right)$ between feed and receiving solution under condition 0 , (b) paracetamol in $A E M\left(\Delta \mathrm{C}_{0}=0.1\right.$, $\left.1,2 \mathrm{~g} \mathrm{~L}^{-1}\right)$ in receiving solution under conditions 0 and $\mathrm{FR}$, (c) clofibric acid in $\mathrm{AEM}\left(\Delta \mathrm{C}_{0}=0.1 \mathrm{~g} \mathrm{~L}^{-1}\right)$, and (d) atenolol in CEM ( $\left.\Delta \mathrm{C}_{0}=0.1 \mathrm{~g} \mathrm{~L}-1\right)$ between feed and receiving solution under conditions 0 and FR. $m$ is the slope of the linear fit. The transport of clofibric acid in CEM and atenolol in AEM was below $1 \%$ and, thus, is not shown.

electroneutrality principle and Donnan Dialysis (Mulder, 2012), either positively-charged ions would transport in the same direction, or $\mathrm{OH}^{-}$would transport in the opposite direction. The change of $\mathrm{pH}$ in the feed (from 6.4 to 7.1 ) and receiving solution (from 7.2 to 6.8) (Fig. S5a) confirmed the latter case since an IEM is not supposed to permeate co-ions. The stability of $\mathrm{pH}$ after $5 \mathrm{~h}$ indicated an electrochemical potential equilibrium across the membrane, corresponding to the observed plateau. No water transport was observed due to the negligible osmotic pressure.

A similar non-linear transport profile was observed for the positively-charged atenolol in CEM (Fig. 3d), where a plateau of $7.7 \mathrm{mg} \mathrm{L} \mathrm{L}^{-1}$ (i.e., far from equilibrium if solely diffusion-driven) was reached after $24 \mathrm{~h}$, indicating a key role of electromigration aside from diffusion. The radius of atenolol $(0.45 \mathrm{~nm})$ would only allow its transport through 0 - $\mathrm{Ps}_{\text {long }}$ free-volume elements. The trend of $\mathrm{pH}$ change of atenolol was opposite to that of clofibric acid, decreasing from 6.2 to 4.9 due to the transport of $\mathrm{H}^{+}$ from the receiving to the feed solution to balance the transported atenolol from the feed (Fig. S5b). However, a significant decrease in the concentration of atenolol in the feed caused by adsorption was observed. During control experiments, atenolol showed high adsorption in CEMs even after consecutive saturation steps (Fig. S1). The considerable adsorption of positively-charged solutes in CEM has been previously observed in membrane-stack studies by Roman et al. (2019).

The transport of paracetamol and clofibric acid in AEM was higher than that of paracetamol and atenolol in CEM (Fig. 3a, $3 c$, and $3 d$ ), again, possibly due to the three reasons as previously suggested and the lower permselectivity of AEM. These results are in agreement with a previous study where the transport of non-charged organic solutes (i.e., paracetamol, theophylline, and phenazone) in two different commercial types of AEMs was higher than in CEMs (Ma et al., 2018). Moreover, until equilibrium was achieved, the transport rate of charged organic solutes was higher than non-charged solutes in the same type of membranes, requiring further investigation. The transport of clofibric acid in CEM and atenolol in AEM was below $1 \%$ due to repulsive electrostatic interactions. Overall, unlike pure diffusion of non-charged organic solutes, both diffusion and electromigration played a key role during the transport of charged solutes.

\subsubsection{Presence of an equally high concentration of salts in solution} in both compartments of the diffusion cell

The transport of organic solutes was studied in the presence of an equally high concentration of $\mathrm{NaCl}\left(29.2 \mathrm{~g} \mathrm{~L}^{-1}\right)$ in solution in both compartments of the diffusion cell (i.e., condition FR). No osmotic pressure difference over the membrane was generated. Also, no water transport was observed during the whole experiment. Paracetamol $\left(\Delta C_{0}=0.1,1\right.$, and $\left.2 \mathrm{~g} \mathrm{~L}^{-1}\right)$ transport across the AEM was again purely diffusive, irrespective of the initial paracetamol concentration gradient (Fig. 3b). However, the presence of salts slightly hindered the transport of paracetamol in AEMs, resulting in lower permeability constant $\left(B_{0}\right.$ and $B_{F R}$ were $4.0 \times 10^{-7} \mathrm{~m} \mathrm{~s}^{-1}$ and $3.2 \times 10^{-7} \mathrm{~m} \mathrm{~s}^{-1}$, respectively). Organic solute diffusivity in the bulk solution is shown to decrease with increasing salinity (Table S1); as such, also the solute membrane permeability constant considerably decreased with an increase in salinity. For paracetamol, the decrease in the diffusion coefficient in the salty solution was $4 \%$, whereas the paracetamol membrane permeability constants decreased by $19 \%$. The permeability constant accounts for solute bulk diffusivity, partitioning into, and hindrance in the membrane phase. Therefore, a large part of this decrease is attributed to a decrease in partitioning and/or an increase in hindrance in the membrane phase. As is shown in section S6, organic solutes were desorbed from both AEMs and CEMs when the membrane was exposed to increased salinity, indicating that the presence of salt ions either resulted in an increased ion exchange (e.g., in the case of atenolol, atenolol ions being exchanged by the $\mathrm{Na}^{+}$ 

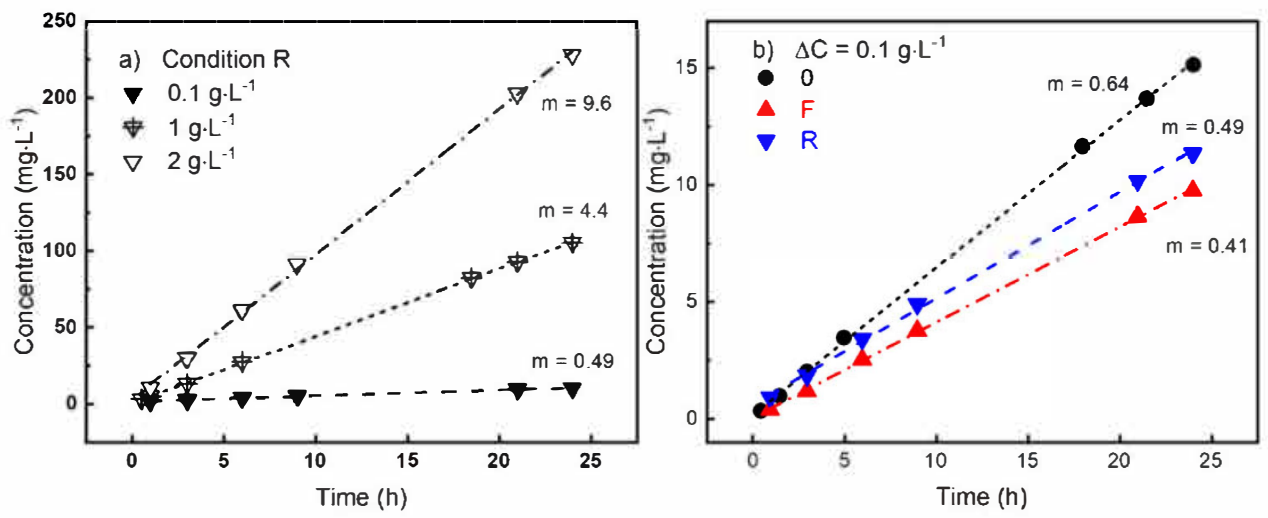

Fig. 4. Concentration profile of paracetamol in the receiving solution at (a) $\Delta C_{0}=0.1,1,2 \mathrm{~g} \mathrm{~L}^{-1}$ in $A E M$ under condition $R$ and (b) $\Delta C_{0}=0.1 \mathrm{~g} L^{-1}$ in $A E M$ under conditions $0, F$, and $R . m$ is the slope of the linear fit.

due to the increased concentration of $\mathrm{Na}^{+}$), and/or decreased the membrane-organic solute adsorption affinity (Fig. S2 and S4).

Likewise, the presence of a high concentration of salts in both compartments also inhibited the transport of clofibric acid in AEM (Fig. 3c) and atenolol in CEM (Fig. 3d). Remarkably, under condition $\mathrm{FR}$, the mass balance of atenolol in the feed and receiving solution slightly varied during the $24 \mathrm{~h}$ of experiments (i.e., within 5\%), in contrast to the adsorption observed during condition 0. Small $\mathrm{Na}^{+}$ions at high concentrations would preferentially interact with the CEMs functional groups; thus, preventing the interactions (i.e., adsorption) of larger and more complex molecules (e.g., organic solutes) (discussed in section S6, Fig. S2-4). This ion-exchange phenomenon was also well observed in negatively-charged forward osmosis membrane using $\mathrm{Na}^{+}$ions to extract $\mathrm{NH}_{4}{ }^{+}$ions, despite that the fluxes of the ions are 3-5 order of magnitudes higher than the charged organic solutes in this study (Lu et al., 2014). Other studies have shown that $\mathrm{NaCl}$ can be used to improve the desorption (cleaning) of organic solutes from IEMs and polymers (Bdiri et al., 2018; Landry and Boyer, 2013).

\subsection{Transport of organic solutes under the influence of a salt gradient}

\subsubsection{Non-charged organic solutes}

The influence of the salt concentration gradient on the transport of paracetamol in IEMs was investigated (i.e., conditions $F$ and $\mathrm{R}$ ). In condition $\mathrm{F}, \mathrm{NaCl}$ was dosed in the feed and receiving solutions at concentrations of $29.2 \mathrm{~g} \mathrm{~L}^{-1}$ and $0.1 \mathrm{~g} \mathrm{~L}^{-1}$, respectively, and vice versa for condition R. Due to the concentration gradient, $\mathrm{NaCl}$ would transport in the same direction of the organic solutes in condition $\mathrm{F}$ (i.e., from feed to receiving solution, or co-transport), while the transport would be expected to occur in the opposite direction in condition $\mathrm{R}$ (i.e., from receiving solution, or counter-transport). At the same time, water and organic solutes would move in the opposite direction in condition $\mathrm{F}$, whereas they would move in the same direction in condition R. A range of $\Delta \mathrm{C}_{0}$ of paracetamol (i.e., $0.1,1$, and $2 \mathrm{~g} \mathrm{~L}^{-1}$ ) was applied in the feed compartment using AEM under condition F and R (Fig. 4a and $4 \mathrm{~b}$ ), yielding a linear concentration increase in all cases, suggesting diffusion as the main driving force of non-charged organic solutes under a salt gradient. The permeability constant of paracetamol for the three conditions followed the order: $\mathrm{B}_{0}\left(4.0 \times 10^{-7} \mathrm{~m} \mathrm{~s}^{-1}\right)>$ $\mathrm{B}_{\mathrm{R}}\left(3.4 \times 10^{-7} \mathrm{~m} \mathrm{~s}^{-1}\right)>\mathrm{B}_{\mathrm{F}}\left(2.7 \times 10^{-7} \mathrm{~m} \mathrm{~s}^{-1}\right)$ (Fig. 5a), indicating that the presence of salts inhibited the transport of paracetamol in AEM regardless of the direction of salt transport. The concentration profile of paracetamol in CEM was also linear; however, the influence of the direction of the transport of salts was different. Briefly, the permeability constant of paracetamol in CEM followed the order: $B_{R}\left(4.7 \times 10^{-8} \mathrm{~m} \mathrm{~s}^{-1}\right)>B_{0}\left(3.2 \times 10^{-8} \mathrm{~m} \mathrm{~s}^{-1}\right)>B_{F}$ $\left(2.3 \times 10^{-8} \mathrm{~m} \mathrm{~s}^{-1}\right)$ (Fig. 5b).

The co/counter- transport of water (i.e., from feed to receiving solution in condition $\mathrm{R}$ and from receiving solution to feed in condition $F$, respectively) were measured (Fig. S6). Due to the same salt concentration gradient, the same osmotic pressure difference over the membrane was generated for conditions $F$ and $R$. Less than $5 \%$ (approximately $7.5 \mathrm{~mL}$ ) of water was transported for both AEM and CEM during the experiments, confirming a similar water permeability of both IEMs (Section 3.1). The osmotic pressure generated by the organic solutes at the concentrations tested was considerably lower than that generated by the salt gradient and, therefore, can be neglected.

The transport of paracetamol in IEMs under salinity gradients was driven by its concentration gradient and was influenced further by steric hindrance interactions with water and salt ions, with the water and salt transport occurring in opposite directions. PALS results suggest that small salt ions could easily transport through both types of membrane pores irrespective of the influence of organics transport. However, in condition F, due to its larger size, paracetamol could transport through the larger freevolume elements $\left(0-\mathrm{Ps}_{\text {long }}\right)$, while being hindered by small salt ions in the smaller free-volume elements $\left(0-\mathrm{Ps}_{\text {short}}\right.$, which are much more abundant than its counterpart in the same type of the membranes, indicating an easier and higher transport of salt ions). The hindrance of the co-transport of salts to the transport of organic solutes has been suggested for ED processes. Salt ions are strong competitors in transport due to their smaller size, higher diffusivity, and in this case, higher concentration gradient (Vanoppen et al., 2014). In contrast, the transport of paracetamol in condition $\mathrm{R}$ would be promoted by the co-transport of water, which would drag paracetamol towards the receiving solution through convection.

Notably, for both IEMs, paracetamol was transported significantly less when co-transported with salt and counter-transported with water (condition F) compared to the inverse case (condition $\mathrm{R}$ ), confirming the inhibition by salts and occurrence of convection, respectively. From a steric point of view, this would suggest that steric hindrance interactions between paracetamol and $\mathrm{NaCl}$ are much smaller than those with water. This implies that the diffusivity of organic solutes would show low sensitivity to solution salinity, which was confirmed in this study: organic solute diffusivity decreased by about $5 \%$ when salinity increased from 0 to $0.5 \mathrm{M}$, without correcting for increased solution viscosity (Table S1). Similarly, D'Haese (2019) showed that after correcting for increasing viscosity at increasing salinity, the diffusivity of atenolol was almost constant, while salinity varied between 0 and $4 \mathrm{M} \mathrm{NaCl}$. However, this steric hindrance hypothesis alone fails to explain the 


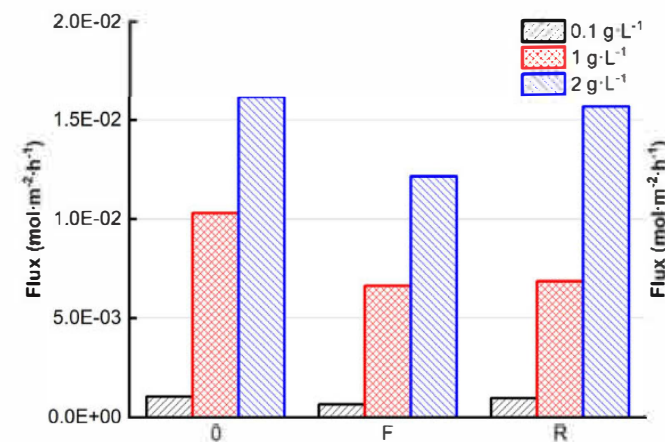

a) AEM

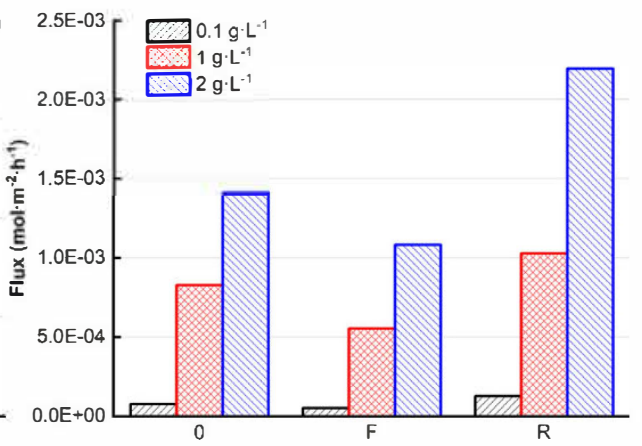

b) CEM

Fig. 5. Average flux of paracetamol at $\Delta \mathrm{C}_{0}$ of $0.1,1$, and $2 \mathrm{~g} \mathrm{~L}^{-1}$ under conditions $0, \mathrm{~F}$, and $\mathrm{R}$ in (a) AEM and (b) CEM.
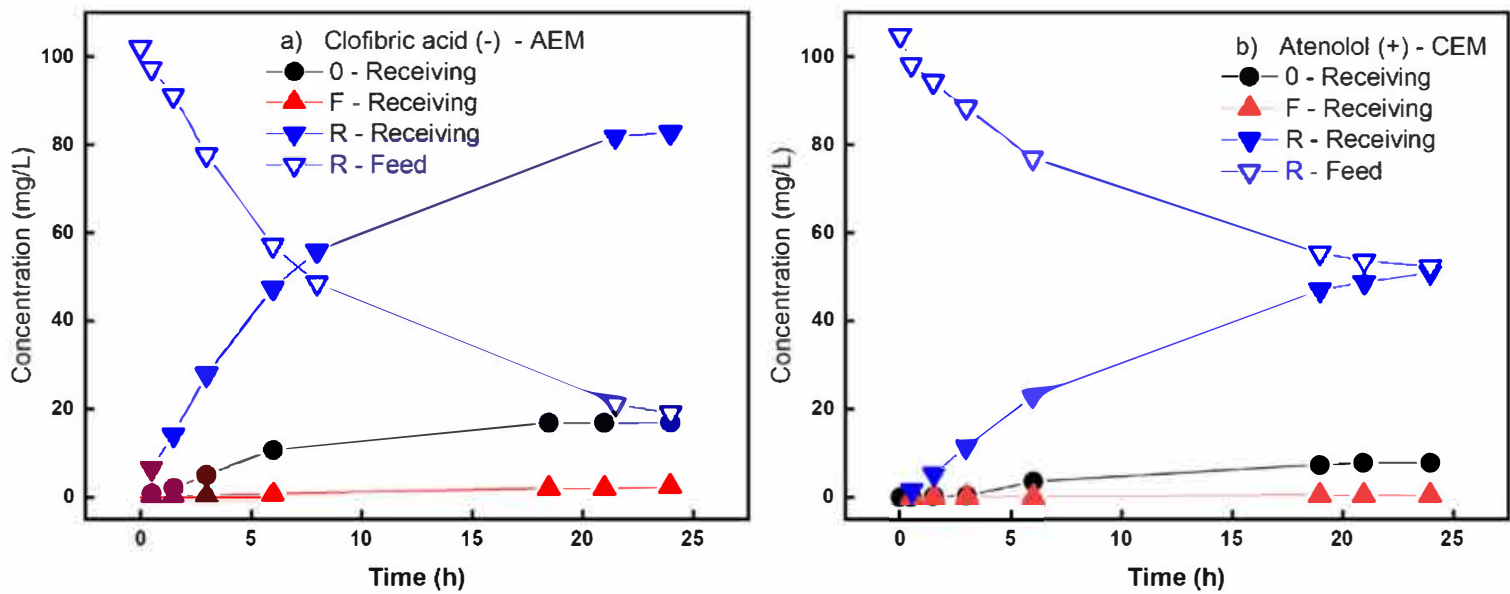

Fig. 6. Concentration profile of (a) clofibric acid in AEM and (b) atenolol in CEM at $\Delta C_{0}=0.1 \mathrm{~g} \mathrm{~L}^{-1}$ in the feed under condition $\mathrm{R}$ and in the receiving solution under conditions $0, F$, and $R$.

discrepancy between AEM and CEM when comparing cases 0 and $\mathrm{R}$ : an opposing salinity gradient appeared to promote paracetamol transport in the CEM membrane while the reverse was true in the AEM membrane. Thermodynamic considerations, such as the influence of salinity on interfacial interactions between organic solutes and the membrane at both interfaces and solute activity gradients within the membrane, will need to be included.

Overall, the transport of salts inhibited the transport of noncharged organic solutes, while the transport of water triggered the convective transport of the solutes. Although convection played a role in the overall transport, diffusion would be the dominant transport mechanism of non-charged organic solutes in the presence of salt gradient.

\subsubsection{Charged organic solutes}

The influence of the salt concentration gradient on the transport of charged organic solutes in IEMs was investigated (i.e., conditions $\mathrm{F}$ and R) (Figs. 6 and 7). Both clofibric acid and atenolol showed the same order in transport behavior: condition $R>$ condition $0>$ condition $F$. In condition $R$ (i.e., counter-transport of salts), ion exchange took place between negatively-charged clofibric acid and $\mathrm{Cl}^{-}$in AEMs and between positively-charged atenolol and $\mathrm{Na}^{+}$ in CEMs. $\mathrm{NaCl}$ was present in the receiving compartment at a considerably higher molar concentration than either organic solute. As a result, the concentration-driven transport of $\mathrm{Cl}^{-}$caused an electrostatic potential gradient over the membrane, further in favor of the uphill transport of clofibric acid (transported mass $74 \%$ after $24 \mathrm{~h}$ ). Moreover, this uphill transport occurred until an equilibrium was reached, where the electrochemical potential gradi-

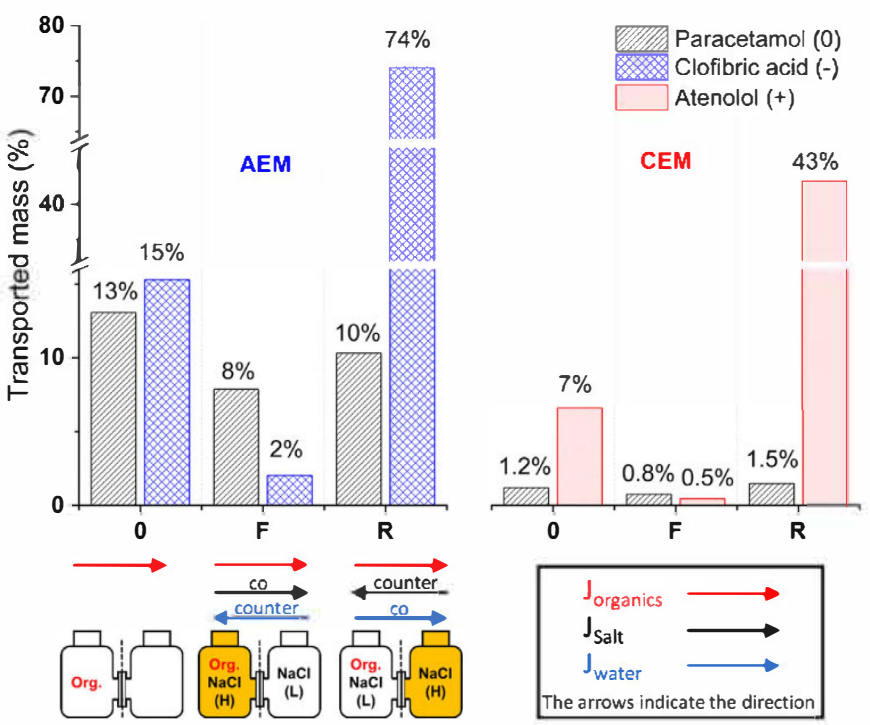

Fig. 7. Transported mass of organic solutes (\%) under conditions $0, F$, and $R$ in AEM and $\operatorname{CEM}\left(\Delta \mathrm{C}_{0}=0.1 \mathrm{~g} \mathrm{~L}^{-1}\right)$.

ent of all ions and charged solutes is zero between compartments (Post et al., 2009). Although the transport of atenolol reached $43 \%$ after $24 \mathrm{~h}$, the trend shows a gradual increase over the equilibrium level. In condition $\mathrm{F}, \mathrm{NaCl}$ permeation inhibited the transport of organic solutes (less transport than in condition 0 ), due to $\mathrm{Na}^{+}$ 
and $\mathrm{Cl}^{-}$having a higher diffusivity in AEM and CEM compared to clofibric acid and atenolol, respectively. Additionally, AEM is more permeable to clofibric acid than CEM is to atenolol. This can be related to the larger size of atenolol and the smaller $0-\mathrm{Ps}_{\text {long }}$ freevolume elements of CEM compared to AEM (Section 3.2.1).

The contribution of convective drag by water to the transport of charged organic solutes is likely to be minimal. The difference in transported mass between condition $\mathrm{R}$ and $\mathrm{F}$ for both charged organic solutes is large (differing by 1-2 orders of magnitude), while the corresponding difference for paracetamol is less than twofold (Fig. 7). This is to be expected, given that low water permeability is a design criterion for IEMs, combined with decoupling of fluxes in dense polymer matrices.

A schematic overview was proposed to show the transport of ions (Fig. S7). Briefly, in condition $\mathrm{R}$ and based on salt gradient, $\mathrm{Cl}^{-}$were continuously transported through AEM from the receiving to the feed solution through both the larger and (more abundant) smaller free-volume elements. To maintain electroneutrality, both negatively-charged clofibric acid and $\mathrm{OH}^{-}$were transported in the opposite direction, causing a drop of $0.7 \mathrm{pH}$ units in the feed and an increase of $0.3 \mathrm{pH}$ units in the receiving solution (Fig. S8a). Similarly, the transport of $\mathrm{Na}^{+}$in CEM from receiving solution to the feed induced the transport of positively-charged atenolol and $\mathrm{H}^{+}$in the opposite direction to maintain electroneutrality; thus, showing an increase of $0.8 \mathrm{pH}$ units in the feed (Fig. $\mathrm{S} 8 \mathrm{~b}$ ). Roman et al. (2019) explained the promotion of charged organic solutes by the significantly higher concentration of salt ions by the thermodynamic equilibrium of the system following Donnan Dialysis. Therefore, the electrostatic potential gradient brought by a high salt concentration gradient, which is transported against the charged organic solutes gradient (condition $R$ ), is the essential driving force of the latter, promoting the electromigration of charged solutes in IEMs.

In condition $\mathrm{F}$, due to their smaller size and higher concentration gradient, $\mathrm{Cl}^{-}$and $\mathrm{Na}^{+}$transported faster from the feed side to the receiving solution taking up two classes of free-volume elements, inhibiting the transport of clofibric acid ions and atenolol ions through the larger $0-\mathrm{Ps}_{\text {Iong }}$ free-volume elements, respectively. To reach electrochemical potential equilibrium, the transport of $\mathrm{OH}^{-}$and $\mathrm{H}^{+}$in the opposite direction was triggered, resulting in a higher and lower $\mathrm{pH}$ in the feed after $24 \mathrm{~h}$, respectively (Fig. S7 and S8). However, in condition F, the transport direction of clofibric acid and atenolol driven by concentration gradient was against the electrostatic potential generated by the co-transport of salts. Thus, the electromigration triggered by the tendency of equilibrium as in condition $\mathrm{R}$ was significantly limited, while the diffusion driven by its concentration gradient was also affected. Hence, in condition F, the diffusion of charged organic solutes was inhibited, while electromigration was highly limited by the co-transport of salts.

In summary, in the presence of a salt gradient, the concentration gradient of organic solutes was the main driving force for noncharged organic solutes transport (diffusion). Conversely, the electrostatic potential gradient considerably influenced the transport of charged organics. The co-transport of salts inhibited the transport of charged organics (mainly electromigration), while countertransport of salts promoted the latter (mainly diffusion). Although water transport (convention) played a role for non-charged organic solutes transport, it was less critical for charged solutes due to other dominant driving force (electrostatic potential gradient).

\subsubsection{Practical implications and future perspective}

IEM-based technologies enable the separation of organic and inorganic species in complex streams and further resource recovery (Nagarale et al., 2006). Bioenergy technologies (i.e., microbial fuel and electrolysis cells) using IEMs are key in producing biofuels or valuable chemicals from complex industrial wastewaters containing organic and inorganic pollutants (Zhang and Angelidaki, 2014). Together with emerging OMPs in municipal wastewater, these circumstances raised concern regarding organic solutes/OMPs transport through IEMs, resulting in the contamination of the product streams (Arola et al., 2019; Pronk et al., 2006; Vanoppen et al., 2014). The results of this study provided key implications for IEMs-based processes dealing with complex streams and further resource recovery. Specifically, the co-existence of organic solutes/OMPs and inorganic salts (condition F) would result in an inhibition of OMPs transport and lower contamination of the product streams. For the opposite case, e.g., RED processes for drinking water production, the organic solutes/OMPs can be transported from the impaired wastewater to the seawater/brine compartment (condition R, counter-transport of salts); thus, posing an added risk to the drinking water source (i.e., promoting the transport of OMPs). In the presence of an external current supply, however, the dominant transport mechanisms of salts and organic solutes would potentially differ from those reported in the current study. Nevertheless, whether the electric potential is applied using an external power source, or imposed by salt diffusion through permselective membranes, the resulting electrostatic driving force for the transport of organic solutes is identical. Moreover, the behavior of organic solutes is also governed by the properties of the solutes and the membranes; consequently, the current results are specific for the set of membranes and solutes used and do not necessarily apply to any combination of OMP and IEM. Nevertheless, the trends observed with respect to the effect of the presence of a salt gradient are valid across membranes and solutes, as confirmed by the vast literature with inorganic solutes. Finally, by analyzing free-volume elements using the PALS technique, high selective separation and low defects can be achieved for next-generation IEMs.

\section{Conclusions}

The transport mechanisms of non-charged and charged organic solutes in IEMs were investigated under the influence of a) the presence, b) concentration gradient, and c) transport direction of salts. The overall transport of organic solutes can be described by the same mechanisms that have been shown to describe the transport of inorganic solutes (i.e., diffusion, convection, and electromigration). In the absence and the presence of an equal concentration of salts, the transport of non-charged organic solutes was solely driven by diffusion, while both diffusion and electromigration dominated the transport of charged organic solutes. A lower transport of organic solutes in the latter condition was caused due to the lower diffusion coefficient of solutes in the presence of salts and the increased hindrance and/or decreased partitioning in the membrane phase. In the presence of a salt gradient, diffusion was the main transport mechanism of non-charged organic solutes, while the counter-transport of salts promoted the transport of charged organics through electromigration. Conversely, the co-transport of salts hindered the transport of organic solutes, where the main transport mechanism was diffusion. Convection played a role in the transport of non-charged organic solutes, while showed minimal to charged solutes due to other dominant driving force (i.e., electrostatic potential gradient). Remarkably, both AEM and CEM showed two classes of free-volume elements from PALS: a smaller 0 -Ps short where salts and smaller organic solutes would be transported, and a larger 0 -Ps organic solutes. The $o-\mathrm{Ps}_{\text {short }}$ class was considerably more abundant than its counterpart; thus, favoring the transport of small salt ions. These results provide key implications in two aspects: 1) the co-existence of organic solutes/OMPs and inorganic salts would result in the inhibition of OMPs transport and lower contamination of the product streams in ED processes), and 2) the organic solutes/OMPs can be transported from impaired wastewater to the 
seawater/brine compartment; thus, posing an added risk to the drinking water source in RED processes.

\section{Declaration of Competing Interest}

The authors declare that they have no known competing financial interests or personal relationships that could have appeared to influence the work reported in this paper.

\section{Acknowledgments}

The financial support provided by the China Scholarship Council (File No. 201406430009) and the scientific review of the manuscript by Dr. Mingsheng Jia are acknowledged. PALS experiments were performed at the NEPOMUC PLEPS instrument operated by FRM-II at the Heinz Maier Leibnitz Zentrum (MLZ) in Garching, Germany. The $500 \mathrm{MHz}$ NMR equipment was funded by the Hercules Foundation (contract number: AUGE09/006). NMR measurements were performed at the UGent NMR Centre by Dr. Lorenz De Neve.

\section{Supplementary materials}

Supplementary material associated with this article can be found, in the online version, at doi:10.1016/j.watres.2020.116756.

\section{References}

Al-Amshawee, S., Yunus, M.Y.B.M., Azoddein, A.A.M., Hassell, D.G., Dakhil, I.H., Hasan, H.A, 2020. Electrodialysis desalination for water and wastewater: a review. Chem. Eng. J. 380, 122231.

Algers, J. Sperr, P., Egger, W. Kögel, G., Maurer, F.H.J. 2003. Median implantation depth and implantation profile of 3-18 keV positrons in amorphous polymers. Phys. Rev. B 67 (12).

Arola, K., Ward, A., Mänttäri, M., Kallioinen, M., Batstone, D, 2019. Transport of pharmaceuticals during electrodialysis treatment of wastewater. Water Res. 161 496-504.

Bdiri, M., Dammak, L., Chaabane, L., Larchet, C., Hellal, F., Nikonenko, V., Pismenskaya, N.D, 2018. Cleaning of cation-exchange membranes used in electrodialysis for food industry by chemical solutions. Sep. Purif. Technol. 199, 114-123.

Campione, A., Gurreri, L., Ciofalo, M., Micale, G., Tamburini, A., Cipollina, A., 2018 Electrodialysis for water desalination: a critical assessment of recent developments on process fundamentals, models and applications. Desalination 434, 121-160.

Chen, Y., He, H., Liu, H., Li, H., Zeng, G., Xia, X., Yang, C., 2018. Effect of salinity on removal performance and activated sludge characteristics in sequencing batch reactors. Bioresour. Technol. 249, 890-899.

D'Haese, A.K.H., 2019. Interactions between feed solutes and inorganic electrolytic draw solutes in forward osmosis. J. Memb. Sci., 117636

Długołęcki, P., Nymeijer, K., Metz, S., Wessling, M., 2008. Current status of ion exchange membranes for power generation from salinity gradients. J. Memb. Sci. $319(1-2), 214-222$.

Egger, W., Sperr, P., Kögel, G., Dollinger, G., 2007. Pulsed low energy positron system (PLEPS) at the Munich research reactor FRM II. Phys. Status Solidi (c) 4 (10) 3969-3972.

Fan, H. Yip, N.Y., 2019. Elucidating conductivity-permselectivity tradeoffs in elec trodialysis and reverse electrodialysis by structure-property analysis of ion-exchange membranes. J. Memb. Sci. 573, 668-681.

Gonzalez, A., Grágeda, M., Ushak, S., 2017. Assessment of pilot-scale water purification module with electrodialysis technology and solar energy. Appl. Energy 206, 1643-1652.

Grandclement, C., Seyssieç, I., Piram, A., Wong-Wah-Chung, P., Vanot, G., Tilia$\cos$, N., Roche, N., Doumenq, P., 2017. From the conventional biological wastewater treatment to hybrid processes, the evaluation of organic micropollutant removal: a review. Water Res. 111, 297-317.

Gugliuzza, A., 2015. In: Drioli, E., Giorno, L. (Eds.). Springer Berlin Heidelberg, Berlin, Heidelberg, pp. 1-2.

Han, L., Galier, S., Roux-de Balmann, H., 2016. Transfer of neutral organic solutes during desalination by electrodialysis: influence of the salt composition. J. Memb. Sci. 511, 207-218.

Hayduk, W., Laudie, H., 1974. Prediction of diffusion coefficients for nonelectrolytes in dilute aqueous solutions. AIChE J. 20 (3), 611-615.
Hugenschmidt, C., Dollinger, G., Egger, W., Kögel, G., Löwe, B., Mayer, J., Pikart, P., Piochacz, C., Repper, R., Schreckenbach, K., Sperr, P., Stadlbauer, M., 2008. Surface and bulk investigations at the high intensity positron beam facility NEPOMUC. Appl. Surf. Sci. 255 (1), 29-32.

Hugenschmidt, C., Piochacz, C., Reiner, M., Schreckenbach, K., 2012. The NEPOMUC upgradeand advanced positron beam experiments. New J. Phys. 14 (5), 055027.

Kingsbury, R.S., Coronell, O., 2020. Modeling and validation of concentration dependence of ion exchange membrane permselectivity: significance of convection and Manning's counter-ion condensation theory. J. Memb. Sci, 118411.

Kirkegaard, P., Olsen, J.V., Eldrup, M.M., 2017. PALSfit3: A Software Package For Analysing Positron Lifetime Spectra. Technical University of Denmark, Kgs. Lyngby.

Landry, K.A., Boyer, T.H., 2013. Diclofenac removal in urine using strong-base anion exchange polymer resins. Water Res. 47 (17), 6432-6444.

Lefebvre, O., Moletta, R., 2006. Treatment of organic pollution in industrial saline wastewater: a literature review. Water Res. 40 (20), 3671-3682.

Li, W., Krantz, W.B., Cornelissen, E.R., Post, J.W., Verliefde, A.R.D., Tang C.Y, 2013. A novel hybrid process of reverse electrodialysis and reverse osmosis for low energy seawater desalination and brine management. Appl. Energy 104, 592-602.

Lu, X., Boo, C., Ma, J., Elimelech, M., 2014. Bidirectional diffusion of ammonium and sodium cations in forward osmosis: role of membrane active layer surface chemistry and charge. Environ. Sci. Technol. 48 (24), 14369-14376.

Ma, L., Gutierrez, L., Vanoppen, M., Lorenz, D.N., Aubry, C., Verliefde, A., 2018. Transport of uncharged organics in ion-exchange membranes: experimental validation of the solution-diffusion model. J. Memb. Sci. 564, 773-781.

Mulder, M., 2012. Basic Principles of Membrane Technology. Kluwer Academic Publishers, Dordrecht, The Netherlands.

Nagarale, R.K., Gohil, G.S., Shahi, V.K., 2006. Recent developments on ion-exchange membranes and electro-membrane processes. Adv. Colloid Interface Sci. 119 $(2-3), 97-130$.

Nam, J.-Y., Hwang, K.-S., Kim, H.-.C., Jeong, H., Kim, H., Jwa, E., Yang, S., Choi, J. Kim, C.-S. Han, J-H. Jeong, N. 2019. Assessing the behavior of the feed-water constituents of a pilot-scale 1000-cell-pair reverse electrodialysis with seawater and municipal wastewater effluent. Water Res. 148, 261-271.

Nghiem, L.D., Schäfer, A.I., Elimelech, M., 2006. Role of electrostatic interactions in the retention of pharmaceutically active contaminants by a loose nanofiltration membrane. J. Memb. Sci. 286 (1), 52-59.

Post, J.W., Hamelers, H.V.M., Buisman, C.J.N, 2009. Influence of multivalent ions on power production from mixing salt and fresh water with a reverse electrodialysis system. J. Memb. Sci. 330 (1-2), 65-72.

Pramanik, B.K., Nghiem, L.D., Hai, F.I., 2020. Extraction of strategically important elements from brines: constraints and opportunities. Water Res. 168, 115149.

Pronk, W., Biebow, M., Boller, M., 2006. Electrodialysis for recovering salts from a urine solution containing micropollutants. Environ. Sci. Technol. 40 (7) 2414-2420.

Roman, M. Van Dijk, LH, Gutierrez, L, Vanoppen, M. Post, JW, Wols, B.A., Cor nelissen, E.R., Verliefde, A.R.D., 2019. Key physicochemical characteristics governing organic micropollutant adsorption and transport in ion-exchange membranes during reverse electrodialysis. Desalination 468, 114084.

Sperr, P., Egger, W. Kögel, G. Dollinger, G., Hugenschmidt, C., Repper, R., Piochacz, C. 2008. Status of the pulsed low energy positron beam system (PLEPS) at the Munich Research Reactor FRM-II. Appl. Surf. Sci. 255 (1), 35-38.

Strathmann, H., 2004. Ion-Exchange Membrane Separation Processes. Elsevier.

Strathmann, H., 2010. Electrodialysis, a mature technology with a multitude of new applications. Desalination 264 (3), 268-288.

Tedesco, M., Hamelers, H.V.M., Biesheuvel, P.M, 2016. Nernst-Planck transport theory for (reverse) electrodialysis: I. Effect of co-ion transport through the membranes. J. Memb. Sci. 510, 370-381.

Tedesco, M., Hamelers, H.V.M., Biesheuvel, P.M, 2017. Nernst-Planck transport theory for (reverse) electrodialysis: II. Effect of water transport through ion-exchange membranes. J. Memb. Sci. 531, 172-182.

Vanoppen, M., Bakelants, A.F., Gaublomme, D., Schoutteten, K.V., Bussche, J.V., Vanhaecke, L., Verliefde, A.R., 2014. Properties governing the transport of trace organic contaminants through ion-exchange membranes. Environ. Sci. Technol. 49 (1), 489-497.

Vanoppen, M., van Vooren, T., Gutierrez, L., Roman, M., Croué, L.J.P., Verbeken, K., Philips, J., Verliefde, A.R.D., 2019. Secondary treated domestic wastewater in reverse electrodialysis: what is the best pre-treatment. Sep. Purif. Technol. 218, $25-42$.

Ward, A.J., Arola, K., Thompson Brewster, E., Mehta, C.M., Batstone, D.J., 2018. Nutrient recovery from wastewater through pilot scale electrodialysis. Water Res. 135, 57-65.

Zhang, Q.G., Liu, Q.L., Zhu, A.M., Xiong, Y., Ren, L., 2009. Pervaporation performance of quaternized poly(vinyl alcohol) and its crosslinked membranes for the dehydration of ethanol. J. Memb. Sci. 335 (1), 68-75.

Zhang, Y., Angelidaki, I., 2014. Microbial electrolysis cells turning to be versatile technology: recent advances and future challenges. Water Res. 56, 11-25.

Zhao, D., Lee, L.Y., Ong, S.L., Chowdhury, P., Siah, K.B., Ng, H.Y., 2019. Electrodialysis reversal for industrial reverse osmosis brine treatment. Sep. Purif. Technol. 213, 339-347. 